\title{
Towards multimodal interactions in the multilingual EFL classroom: Lessons from a COIL experience
}

\author{
Sajjad Pouromid \\ Critical Studies in Transformative Education, Graduate School of Human Sciences, Osaka University, \\ 2-1 Yamadaoka, Suita, Osaka, Japan
}

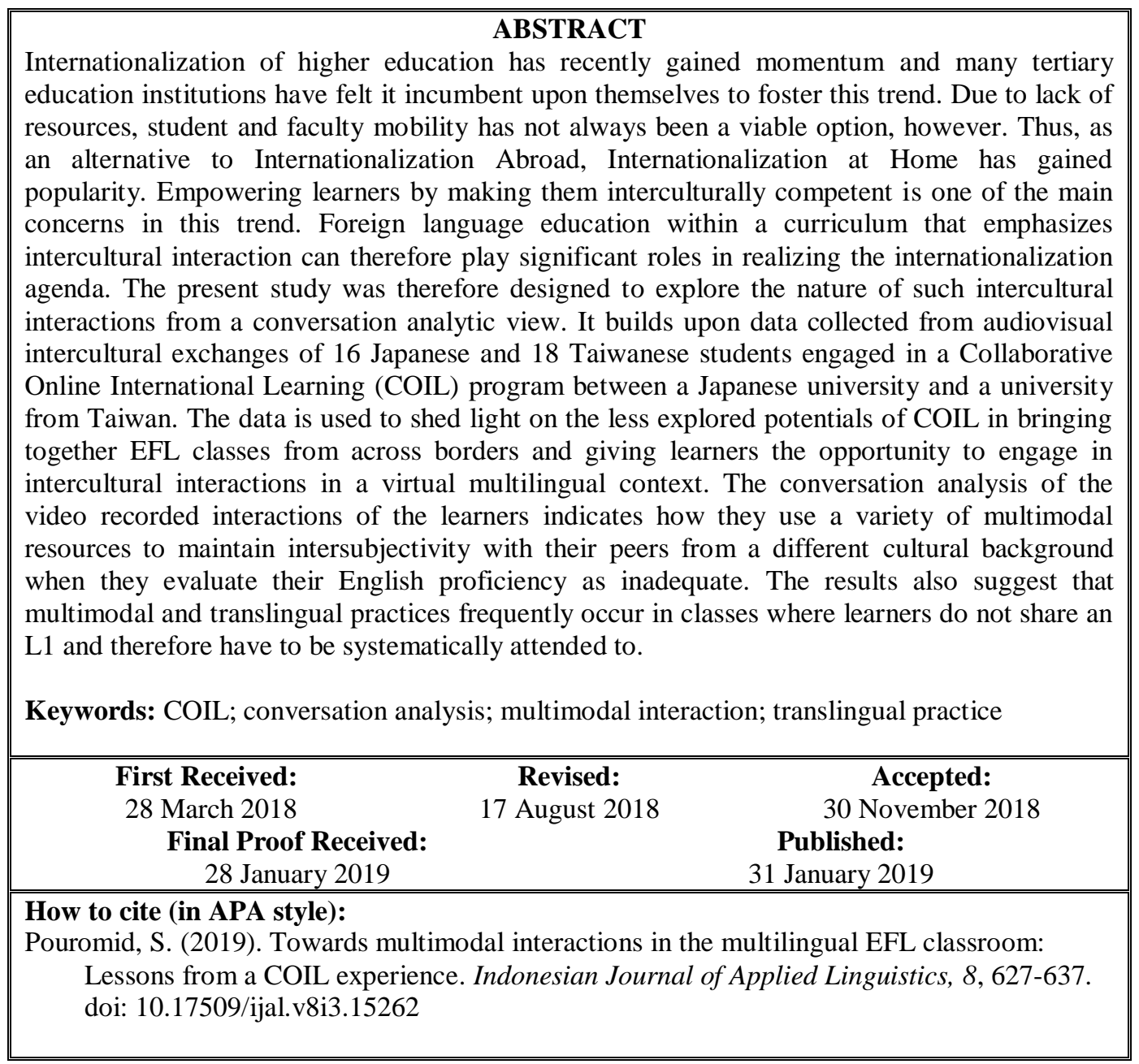

\section{INTRODUCTION}

The increasing demands of globalization have made it necessary for higher education institutions to embark on internationalization. For a higher education institution to survive in a highly competitive market, it has to cater for the growing needs of the situation. One such demand is that universities can no longer depend on domestic students. They need to develop strategies to attract students from overseas as well. Of equal importance is the fact that even domestic students might no longer be merely interested in acquiring domestic level skills. In other words, along with the increasing student mobility prospects and possibilities worldwide, it is understandably expected that universities will prepare students to compete for job opportunities globally. In order to achieve these two goals, universities need to come up with new ways both to become internationally recognized and to enhance the international experience of their faculty, staff, and students. In response to these demands, many universities around the globe have come to recognize their role in promoting international and intercultural abilities in their students (de Wit, 2010). 


\section{Internationalization at home}

Internationalization has taken different forms and hence has been defined in several ways. However, one of the most cited definitions is the one offered by Knight (2004) who states that internationalization is "the process of integrating an international, intercultural, or global dimension into the purpose, functions or delivery of post-secondary education" (p. 11). The definition is particularly interesting because it explicitly denotes internationalization as a process and explicitly refers to international and intercultural aspects of this process. It also seems to be broad in scope encompassing almost any activity at the level of planning or implementation. Knight (2006) further explores the concept of setting distinctions between Internationalization Abroad (IA) and Internationalization at Home ( $\mathrm{IaH})$. IA is characterized by education taking place across borders, the mobility of students, teachers, scholars, programs, courses, curriculum, and projects. On the other hand, $\mathrm{IaH}$ comprises activities designed to help students develop international understanding and intercultural skills. The distinction, however, is not free from controversy.

Beelen and Jones (2015), for instance, call the distinction problematic due to the fact that it implicitly suggests that IA is incapable of developing international and intercultural skills in students and that $\mathrm{IaH}$ is merely done through implementing activities in the absence of a unifying core curriculum. Knight (2006), of course, refers to other factors related to $\mathrm{IaH}$ including the international or intercultural dimensions of the curriculum, research collaboration as well as area and foreign language studies. Her incorporation of curriculum into the idea of $\mathrm{IaH}$ opens up new discussions regarding how this is to be achieved. Responses to this question would vary ranging from proposing solutions through internationalizing curriculum to campus internationalization.

The Organization for Economic Co-operation and Development (OECD, 1996) defines internationalized curriculum as one "with an international orientation in content and/or form, aimed at preparing students for performing (professionally/socially) in an international and multicultural context and designed for domestic and/or foreign students" (OECD, 1996, p. 6). Campus internationalization is also often used interchangeably with comprehensive internationalization as proposed by Hudzik (2011). According to him, comprehensive internationalization is best conceptualized as a commitment realized through action encompassing all aspects of teaching, research, and services in a higher education institution. It is hence not limited to the campus life of students and should be instead extended to the institutions' "external frames of reference, partnerships, and relations" (p. 6).

However IaH is done, it may be tenable to argue that foreign language education, as referred to by Knight (2006), is an inseparable part of it. If the ultimate goal is to enable domestic students to function internationally, it will not be achievable without providing them with the tools do so. However, language instruction, or more particularly speaking, second or foreign language instruction at universities with the aim of producing interculturally competent students will not be free from challenges. For sure, it will take conscious and deliberate efforts to redefine curricula, classrooms, and campuses as common areas that have the potentials to promote intercultural learning among other things (Agnew \& Kahn, 2014). The way this grand goal is to be achieved will be closely interrelated with one's understanding of what a foreign language is and what it takes to teach or learn a foreign language.

\section{Intercultural competence}

Needless to say, the purpose of making learners internationally competent, through the acquisition of a foreign language, leaves no room for defining foreign language learning as the mastery over the underlying system of a given language. The aim, rather, entails the need to enable learners to use a foreign language efficiently in real-world situations. This is reminiscent of the recent propositions in the field of language teaching to promote communicative competence rather than narrowly focusing on linguistic competence. Closely related to this issue is the necessity of cultivating intercultural competence in language learners who would either aspire to move across borders or rather, as is the case with $\mathrm{IaH}$, communicate smoothly with people from other cultures while residing in their own country of origin. In one proposed model for intercultural competence Usó-Juan and MartínezFlor (2006) state that intercultural competence includes both cultural and non-verbal communication skills. They claim that this competence requires knowledge of the target culture as well as the linguistic skills necessary to communicate in a foreign language. For sure, the development of such competence in learners gives them the chance to enjoy IaH. However, one important question remains unanswered: how can language teaching in an $\mathrm{IaH}$ program promote intercultural competence?

With the introduction of technology into the second language classroom, it has become considerably easier to come up with innovative approaches to foster intercultural competence in language learners. Computer technology has now made it possible for learners around the globe to get in touch with each other with relative ease. Advances in Computer Assisted Language Learning (CALL) may well support this claim. Ally (2004) defines the incorporation of technology into learning in general and e-learning in particular as

the use of the internet to access learning materials; to interact with the content, instructor, and other learners; and to obtain support during the learning process, in order to acquire knowledge, to construct personal meaning, and to grow from the learning experience (p. 5).

It seems that CALL in its different forms is capable of meeting most, if not all, of these 
requirements. CALL can take place both in the form of asynchronous and synchronous communications among learners. In other words, learners can get in touch with each other making use of the web space to post data that can be later retrieved by other peers. On the other hand, they can also get in touch via interfaces and interact in real time. Both these techniques have been introduced to language teaching and can for sure offer numerous possibilities. Research, as a matter of fact, has recently found how online interactions among learners can help them acquire collaborative learning behaviors (Nor, Hamat, \& Embi, 2012). One of the ways this can be realized is through the use of Collaborative Online International Learning (COIL) which, to the best of the author's knowledge, is a less explored area in Foreign Language Teaching (FLT).

Collaborative Online International Learning (COIL) In its simplest form, COIL can be defined as a form of online learning involving classes from two or more countries to heighten learners' awareness of intercultural competence (Shiozaki, 2016). Built into the context of IaH, COIL can prove very useful in promoting intercultural competence as well as other components of communicative language ability in learners. Learners in distant areas can be brought together to enjoy cultural exchange and interaction in a shared foreign language. The interaction, of course, has to be planned and principled. The instructors of the two classes need to meet up before the exchange sessions and plan carefully for what they expect the sessions to achieve and design ways to achieve them. COIL exchanges can happen either synchronously or asynchronously. When applicable, the two classes can be held jointly, and the learners can interact via a computer interface in real time. On the other hand, if the time gap between the two countries does not allow a live session, learners can post videos on different video sharing platforms for their peers from the other country.

Such a program may also have challenges for practitioners and program planners regarding how to trace the development of learners. There need to be rigorous methodologies to monitor the learners' potential improvement in the different components of their language ability. If the COIL is supposed to be a medium for the realization of $\mathrm{IaH}$, it has to be able to explain and track student learning. One way to approach this and to study the development of linguistic and intercultural competence is to resort to Conversation Analysis (CA), since it is best equipped to unravel the organization and order of social action and can hence reveal how learners in a given interaction interpret and analyze each other's actions (Seedhouse, 2004) on a turn by turn basis. Each individual learner's contributions can be monitored ideally over time to assess the development in his or her abilities. This is true particularly about longitudinal CA studies, but learner contributions can be monitored even within a single session of intercultural exchange as well.

\section{Conversation analysis}

In its canonical form, CA can be introduced as a methodology for the investigation of naturally-occurring spoken interactions, or to study any two or more people talking together (Have, 2012). CA is a multidisciplinary methodology which has been widely used in many different fields including second and foreign language acquisition. The implications of CA for language teaching are manifold since it is well equipped to reveal the "social organization of natural language-inuse" (Button \& Lee, 1987, p. 2). CA relies on naturally occurring data and in the case of second language classroom, learner interactions can be regarded as authentic and genuine ones taking place. It is particularly relevant to the investigations of intercultural exchanges in $\mathrm{IaH}$ programs because of the value it assigns to the social distribution of knowledge and the social aspects of learning taking place in the classroom (Firth \& Wagner, 2007). In other words, as BarrajaRohan (2011) puts it, from a CA perspective language is no longer regarded as a set of linguistic items, and learners are no longer considered as deficient L2 speakers but rather as novices as well as a social entity trying to come to grips with a new sociocultural environment (p. 480).

In this sense, CA shares the concerns of language teaching researchers who defy behaviorist and innatist perspectives on the grounds that they are unable to appreciate the social aspects of learning a new language. Linear input, output and uptake models take individuals as units of analysis and miss the bigger social aspects of interaction (Kramsch, 2002). Social interaction, as a distinctive human activity, guides the majority of human actions. Therefore, it should be mandatory for foreign language learners to be acquainted with intricacies involved in the social use of the language in a culture possibly totally different from that of their own (Roberts \& Cook, 2009). CA can prove very helpful through enhancing student learning and making explicit what is involved in talk-in-interaction (Seedhouse, 2004).

\section{Translanguaging}

There are a good number of studies recently conducted within a CA framework both in real classroom contexts and in technologically enhanced environments aiming at investigating the development of different language skills in foreign language learners (Kasper \& Wagner, 2011). Among these studies, there are those which have focused on the concept of translanguaging or the way foreign language learners make use of resources other than the target language being learned to communicate meaningfully with their peers (Wagner, 2018). The original idea of learners' translanguaging came from language learners' use of their first language (L1) to maintain intersubjectivity or mutual understanding of the task or topic with other learners. Interestingly, such findings have challenged the monolingual policies in language education which have had a dominant presence in many language classes around the world. Monolingual policies, also called English-only policies, 
have been around for a long time, but their presence was felt even more after the introduction of the Communicative Approach which dominated language teaching classes in the 1970s. According to Pennycook (1994), "as a whole, the Communicative Approach firmly believed the idea that monolingual teaching [excluding L1] with authentic communication in L2 was the best way to learn a language." Within this view, regulations were made with the intention of minimizing or even banning the use of L1 in EFL classes. Cummins (2007), for instance, posits that in many settings "instructional policies are dominated by monolingual instructional principles that are largely unsupported by empirical evidence and inconsistent with current understandings both of how people learn and the functioning of the bilingual and multilingual mind" ( $p$. 222). Cummins's position that banning L1 use is not tenable is supported in various ways (see Turnbull \& Dailey-O'Cain, 2009 for a review) one of which is the facilitative role of translanguaging in promoting meaningful communication among language learners. Translanguaging, or more accurately speaking, translingual practices are not limited to the use of L1, however.

A classic definition of translanguaging provided by some of the pioneers of CA describes the process as recipient design in interaction. Recipient design can be best understood with the various forms an interlocutor in a conversation displays his/her orientation and sensitivity towards the other party who is the coparticipants in the conversation (Sacks, Schegloff \& Jefferson, 1974).The emphasis on the various forms employed by a participant to accommodate the coparticipants indicates that orientation and sensitivity are not necessarily verbal and linguistic (Hawkins, 2018; Canagarajah, 2017). Recent studies have shown that other aspects of communication such as gaze, gesture, body movement, etc. are employed by language learners to keep the meaningful communication with their peers going (Wagner, 2018).

This latter point can be of significance particularly for the focus of the present paper. That is to say, when EFL learners in a classroom share a first language, as is the case with many L2 classes, they may naturally resort to their L1 to compensate for the gaps in their L2 proficiency. In other words, as the literature suggests, L1 functions as a mediatory tool in the L2 classrooms for the language learners who have not yet mastered the target language. However, in multilingual classes where learners do not share a mother tongue, they will have to look for other semiotic mediations to cater to the emerging needs of their communications. Such nonverbal semiotic resources are often referred to as "multimodal resources" in the literature (Kupetz, 2011) and include hand movements, gaze, body orientation, referring among others. In might not be surprising to come across such classes having learners with different nationalities in an English speaking context. However, in non-native English speaking countries, one would expect EFL learners to share an L1. This is not, however, always the case.

As discussed above, the COIL has made it possible for EFL classes to join other classes across national borders with the aim of giving learners the chance to practice intercultural communication. This way, although the learners who are physically present in a class may share an L1, those who join them from another country can be expected to speak a different L1. As a result, in the case of communicative breakdowns between learners due to gaps in their L2 proficiency, resorting to L1 will not be an option, and learners need to opt for non-verbal or multimodal resources to bridge the gap. Although quite a few researchers have investigated the multimodal and translingual resources learners from different L1s use in the foreign language classroom with a CA framework (Mortensen, 2008; Kääntä, 2012), little is known about how learners manage to maintain intersubjectivity or mutual understanding during an online interchange such as what happens in COIL.

Therefore, the present research was intent on addressing this gap by posing the following research question: How do EFL learners resolve communicative breakdowns occurring during online intercultural interactions?

\section{METHOD \\ Research design}

The present study was conducted within an ethnomethodological design which is mainly concerned with understanding how participants make meaning in a social context. It is, in other words, a method of analyzing social order in verbal and nonverbal interactions among participants and how it is created and maintained. Central to this design is the idea that talk, or human social and verbal interaction often accompanied by nonverbal aids, is orderly and therefore the task of the researcher is to unravel the underlying order. CA studies, as detailed before, can be best situated in this design since, through a turn by turn investigations of human interactions in different social settings, they aim at understanding how interlocutors make and maintain order.

Therefore, ethnomethodology, in a broad sense, and CA in particular shape the theoretical framework of this study since they provide an appropriate tool for understanding how social order is produced in interactions and how the participants in an interaction interpret each others' actions, design suitable responses, and consequently establish and create intersubjectivity or mutual understanding despite all the gaps they may have in their L2 linguistic repertoire (Seedhouse, 2004).

\section{Data collection}

The data for this study was collected from a COIL intercultural exchange program between a university in Japan and another university in Taiwan. During this program, two classes focusing on the development of 
oral English skills from the two universities held joint sessions using a video conferencing application. The two instructors had virtually met each other prior to the joint sessions and planned for the content and collaborative tasks. Five joint sessions were held resulting in more than six hours of video recorded data. Besides, the students in the two classes were assigned into mixed nationality groups and were given topics to discuss with their group members outside the classroom. This also resulted in more than ten hours of video recordings which were used as a part of the data for this study as well.

The medium of discussions in both sets of data was English since the learners in the two classes did not share a first language: the Japanese learners spoke Japanese and English, and the Taiwanese learners spoke Mandarin and English. This is often the case with online intercultural exchanges where participants are from different countries and hence have to speak a shared foreign language. There were 16 learners in the Japanese class and 18 learners in the Taiwanese class, all of whom had English proficiency levels of preintermediate and intermediate (roughly equivalent to A2 and $\mathrm{B} 1$ in CEFR) according to their school placement tests. However, not all learners were present throughout the five COIL exchange sessions so the number of participants would actually vary in each session.

CA studies limit themselves to naturally occurring data, which can be defined as human interactions occurring not for research purposes. In this regard, the data used in this study can be regarded as natural too since the COIL sessions were not set up primarily for research purposes and would hence be in place with or without the collection of data. It is, of course, worth mentioning that all participants' consent was sought before the sessions for collecting anonymous data from their interactions. Based on the purpose of each session, the theme of the interactions between the two classes would vary. However, what all the recorded interactions had in common were cross-cultural topics particularly about the two settings of Japan and Taiwan.

\section{Research procedure}

Once collected, the audiovisual data of learner intercultural interactions were transcribed for the analysis. The transcription for CA research is different from transcriptions of observation or interview data since it should not only capture what is said but also how it is said. The transcription system used in this study was the one offered by Jefferson (1984) a summary of which can be found in Appendix A.

What followed the transcription was the identification of instances where a communicative gap or breakdown between learners from the two classes occurred. One prominent feature of interactional segments including a communicative breakdown was the absence of mutual understanding. That is, all moments during which the interlocutors indicated a lack of understanding either due to the language used or unfamiliarity with the topic was counted as an interaction segment with a communicative breakdown. The turns preceding and following the identified gaps were explicated to find out how the participants in the sequence of turns managed to overcome the communicative gap and reorder their interaction featuring recipient design. Of particular interest for this study were the resources used by the learners other than code-switching or the use of L1 since the learners in either of the two classes did not understand the first language of the other class. Such resources would encompass an array of multimodal communication devices such as gaze, gesture, the use of non-words, etc. Therefore, the aim was to study not only the verbal or linguistic aspects of the interactions but also the nonverbal semiotic resources as mentioned above. In other words, the study kept its primary focus on multimodal interactions among the participants.

\section{FINDINGS AND DISCUSSION}

The analysis of the data supported the idea that when EFL learners, engaged in a meaning-focused communication, do not share an L1, they make use of semiotic resources other than the verbal or linguistic encoding of information to make themselves understood. This is achieved in a number of ways but as the literature suggests the term translingual practices may best describe the strategies employed by EFL learners in such situations. In other words, learners go beyond the language they are expected to use either in the form of using a different language or engaging in multimodal practices including the use of gesture, gaze, referring, etc. to resolve the emerging communication difficulties they may experience. This will be illustrated in the following.

Since reporting the results of research employing CA methodology requires thick and detailed descriptions of the audiovisual data, it is impossible to present all instances of multimodal interaction in the data due to the space limit in this research article. Therefore, one episode of such interactions will be presented here accompanied by a detailed transcription as well as stills from the video recorded data available in Appendix B.

In all of the stills provided in this paper, the righthand side image shows the class in Japan, while the class from Taiwan can be seen on the left. In the particular episode reported here one of the learners in the Japan class who has the experience of serving in a Japanese tea ceremony is asked by the teacher to come to the front, sit close to the camera and the screen, and explains the customs involved in a tea ceremony to the learners in the Taiwan class. The ceremony requires both the host and the guest to display decorum through particular gestures. However, in this episode, the Japanese learner (JL) is instructing her peers in the Taiwan class (TLs) how to drink tea only as a guest in the ceremony. As the following extract indicates, she comes across a number of difficulties while trying to make herself understood, possibly due to gaps in her 
English vocabulary repertoire, to describe the moves involved in drinking tea. However, she manages to make herself understood through multimodal interaction. Other participants in this interaction include the Japanese class teacher (JT), the Taiwanese class teacher (TT) and other learners in the Japanese class (JLs) and the Taiwanese class (TL1, TL2). Let us now take a look at the transcription of the episode. All verbal and nonverbal turns taken by the participants have been numbered for the ease of reference, and a guide to the transcription symbols and abbreviations is provided in appendix A. Participant faces are also blurred for privacy reasons in the screenshots (Appendix B).

Episode 1: Describing a tea ceremony

$1 \mathrm{JT}$ : ((asks JL to come to the front)) please come here (3)

$2 \mathrm{JL}$ : ((walks to the front carrying a bottle, sits down and looks at the screen)) my grandmother is tea ceremony and flower arrangement teacher so when I was (2) twenty years old I was $(1)>($ jaja $)<$ twenTY (.) \$twenTEE::N\$ years old

$3 \mathrm{JT}:=$ twelve?

$4 \mathrm{JL}:=>$ twelve $<(($ laughter $))>$ machigatta $<(($ meaning mistaken in Japanese $))$ TWELVE years old

5 TT: ((laughter))

6 TLs: ((laughter))

$7 \mathrm{JL}$ : I was teached about it (.) so (in Japanese)

$8 \mathrm{JT}:=$ so you mean your grandma TAUGHT you $\downarrow$

$9 \mathrm{JL}$ : yeah (.) we first to drink tea we should (.) right hands ((raises her right hand)) pick right hand ((picks up the bottle looks away from the screen to the camera)) on (.) [on my left hand ((puts the bottle on her left hand))

10 TL1: ((puts her right hand on her left hand as if she is holding a bottle too))]

11 TL2: ((hits her right hand on her left hand imitating JL and makes a loud noise))

12 TLs: ((laughter))

13 JLs: ((laughter))

$14 \mathrm{JL}$ : and tu::rn (2) tu::rn ((draws a circle in the air clockwise $))$ right $>$ two $<$ two ((hold two of her fingers up $))$ times[((demonstrates the action by turning the bottle clockwise twice looking at the camera))

15 TL1: ((imitates JL's action looking at screen))]

16 JT: \$right right\$ she's doing the right thing ((probably referring to TL1))

$17 \mathrm{JL}$ : ((smiles, looking at the screen $))$ and drink (.) ((takes the bottle to her lips)) e::to ((etois a filler in Japanese)) drink (.) three times or four times

18 TL1: ((imitates $J L$ 's action looking at screen))

19 JL: a::nd at last we should sound >susususu< ((holds the bottle to her lips as if drinking from it and looks away from the screen to $J T)$ )

$20 \mathrm{JT}$ : make a sound $\downarrow$

$21 \mathrm{JL}:=$ make a sound $(($ looks back at the screen $))($.$) this is called tsuikiri in Japan ((looks away from the screen to JT))$

$22 \mathrm{JT}:=$ which means?

$23 \mathrm{JL}:$ tsui- e:to ${ }^{\circ}$ tsui-((looks away as if thinking $\left.)\right)$ tsui

24 JT: sui? water?

$25 \mathrm{JL}:$ (2) ((shakes her head as if saying no)) TSUI ((drawing the Japanese character for the word tsui on the desk with her finger)) tsuikiri the end

$26 \mathrm{JT}$ : =aha ending drinking $\downarrow$

$27 \mathrm{JL}$ : =yes (1) and by doing this we can (.) explain wish- (.) we:: can finish drinking ((holds the bottle to her lips again and looks at $J T))(1)$ and last e::to ((cleans the rim of the bottle with her hands, looks at JT as if seeking help with the appropriate vocabulary to refer to the rim)) (1) [my lip ((points to the rim of the bottle and looks away from the teacher to the screen))

28 TL1: ((imitates $J L$ 's action cleaning the rim of an imaginary container in her hand))]

29 JL: and clean? ((looks at the teacher as if seeking confirmation))

30 JT: clean [clean

$31 \mathrm{JL}$ : clean] and put ((puts the bottle on the desk in front of her $))$ and (.) bow ((bows))

32 JT: aha

As the detailed information in the double brackets throughout the episode indicates, there were several instances of multimodal interaction among the participants. In turn 1 JT asks JL who has the experience of serving in a Japanese tea ceremony to come to the front of the class and sit closer to the camera and the microphone. Interestingly, however, as can be seen in Screenshot 1, JL walks to the front equipped with a bottle. Carrying a bottle may seem like a random action at first glance, but as the following turns unfold it proves the contrary. As shall be seen in the followings the bottle turns out to be used as a scaffold for bridging the gap in JL's linguistic repertoire. JL seems to have predicted that she would have to switch to another mode (such as gesture) if she failed to put herself across linguistically.

JL starts her turn after sitting down and putting the bottle in front of her on the desk by telling her peers about her background in the tea ceremony. The following few turns (2-6) are devoted to the correction of an error produced by JL. She referred to her age as *twenteen which was followed by JT's corrective feedback. JL notices the correction in turn 4 and utters the correct form emphatically this time which provokes laughter in TT and TLs. Another error occurs in turn 7 when JL says her grandmother *teached her which is followed by JT's corrective recast in turn 8 . The use of these two incorrect words does not seem to hinder the 
flow of communication since it seems that all other participants in the interaction understand what is meant. However, what JT does by providing JL with corrective feedback has interactional significance. JL facilitates the correction of the error by his feedback, and this seems to prevent the potential communicative breakdown that could take place without corrections. In other words, JL preempts JL's switching to a non-linguistic mode or making use of translanguaging by correcting her error linguistically. Not all instances of errors and gaps are treated like this, however.

So far, the two formal errors of JL were corrected by JT on the spot, and therefore there was no need for $\mathrm{JL}$ to resort to a non-verbal resource to compensate for her insufficient linguistic performance. However, a more serious communicative gap appears in turn 9 where JL seems to evaluate her linguistic insufficient repertoire and reaches for the bottle which she had prepared before to supplement her verbal instructions with gestures. In the same turn, JL illustrates how the guests in a tea ceremony should put the tea bowl on their left hand using the bottle instead of the bowl. She does so by looking directly into the camera as Screenshot 2 shows. In light of the way the following turns unfold, it can be argued that these actions, that is reaching for the bottle and looking directly at the camera are preparatory phases for the initiation of a non-verbal mode of interaction.

Apparently, JL's gaze at the camera elicits nonverbal responses from TL1 and TL2 in turns 10 and 11 who imitate JL's action. The two Taiwanese students' turns are reminiscent of what Schegloff and Sacks (1973) call adjacency pairs. According to them, talk occurs in responsive pairs and any first pair part (JL's illustration) necessitates the second pair part (TL1 and TL2's imitation). This is particularly interesting because there is no "talk" actually occurring in the common sense of the term. What has substituted talk here is the multimodal interaction among JL, TL1, and TL2 who have switched the modality from verbal interaction to nonverbal interaction. In more technical terms, what is happening at this moment is what Kress (2003) calls transduction. Through transduction, information coded in one modality (verbal in this case) is restructured into another modality (the use of gestures and nods). TL1 continues to respond to JL's instructions by imitating her in turn 15 , and this is noticed by $\mathrm{JT}$ who confirms her action in turn 16. TL1's second pair part responses continue until turn 18 . The reason why only two of the learners in the Taiwan class respond to JL's first pair parts may lie in the fact that L1 and L2 are closer to the monitor and the camera and may have taken it for granted that $\mathrm{JL}$ is addressing them. Furthermore, in such an interaction where no single TL is nominated by $\mathrm{JL}$ for taking the next turn, any of the TLs may self select the next turn taker, and this obviates the need for other TLs to provide a second pair part.

In turn 19, JL signals a new subtopic by uttering a case opening and with a prolonged vowel. Here she is trying to explain the Japanese custom of tsuikiri which may be best translated into English as the completion slurp. She tries to indicate that the guests at a tea ceremony are expected to slurp their last sip from the tea bowl, but it seems that she cannot find the lexical item needed to describe this.

Instead of searching for the appropriate word, therefore, she switches to another mode and acts it out. In fact, she uses a non-word susususu to refer to the sound produced by the slurp and supplements it by taking the bottle to her lips and acting as if she is sipping from it. At this moment, she looks away from the screen to JT signaling that she needs help with the vocabulary which is responded to by the teacher in turn 20. Of particular interest is the way JL is managing turn allocation and turn-taking at this moment since she signals without uttering a word the participant (her teacher in this case) whom she prefers to take the next turn. It is not uncommon to observe that in the classroom context, where power relations exist, it is the teacher who either selects the next speaker or continues into the next turn. In this extract, however, it is ironically the learner who is selecting the next speaker by nominating him with her gaze.

In turn 21, JL acknowledges JT's help by repeating his words. However, she is still not sure if she has conveyed the meaning and therefore resorts to translanguaging by switching to Japanese as her L1 and uttering the term tsuikiri. What is interesting at this moment is that while translanguaging, JL looks away from the screen to the teacher (Screenshot 3) whom she guesses knows the meaning of the Japanese word. In other words, although she is making use of her L1 in this multilingual context, she seems to be aware of the fact that it cannot be of any help to her Taiwanese peers since they do not understand Japanese. For this reason, she does not orient to the screen or the camera (both in terms of gaze and posture) and instead looks at JT who is living in Japan and is more likely to understand the Japanese word. JL seems to be using both verbal and non-verbal semiotic resources at the same time skillfully deciding in real time when and for whom to use which semiotic medium.

The teacher does not seem to be sure since he has apparently mistaken the word tsui (meaning ending) for sui (meaning water), another word in Japanese with a similar pronunciation (turn 24). At this moment JL quickly comes up with another solution and draws the Japanese pictographic character (kanji) for the word tsui with her finger on the desk hoping that it will help JT understand the word. While doing this, she is still looking at JT since she believes the learners in the other class cannot use her translanguaging hint to make meaning. In turn 26, JT finally understands the referent for tsui and verbalizes the meaning which is quickly confirmed by JL.

A final instance of translingual practice can be seen in turn 27 where JL is trying to explain one of the last moves involved in drinking tea in the ceremony. The guests have to clean their lip mark from the rim of the bowl from which they have drunk tea. To explain 
this, however, seems to be difficult for $\mathrm{JL}$ and the pauses in her turn indicate this. Instead of giving up the idea, however, she makes uses of the bottle once again treating it as a scaffold for putting herself across. She performs the action of cleaning the rim with her hand pointing to it with her finger while keeping an eye on the screen to evaluate TL1's response as can be seen in Screenshots 4 and 5. These actions are in place due to JL's decision to opt for a different mode which can be called acting, showing, or referring in this case. This decision could be motivated by a number of reasons including JL's evaluation of her own linguistic ability or simply her unwillingness to expose her linguistic performance. Either way, as a result of this shift in the mode of interaction, intersubjectivity seems to have been achieved.

JL seems reassured to see her Taiwanese peer imitating her action. At this point, and in turn 29 she comes up with the verb clean and verbalizes it with a rising intonation at the same time looking away from the screen to the teacher once again. This look which serves the function of nominating JT for the next turn elicits a confirmation response from the teacher in turn 30. JL then ends the episode by performing a bow as the final move in the ceremony which is followed by JT's second pair part contribution with a case closing aha.

\section{CONCLUSION}

The EFL scene has undergone changes in different regards throughout the few past decades. One such change is the way foreign language teaching is perceived now as a means of cultivating skills other than linguistic ones in learners to enable them to function competently in higher education. The increasing demands of globalization have also made it necessary for higher education institutions to pursue internationalization in order to remain in an increasingly competitive market by introducing $\mathrm{IaH}$ programs. Foreign language pedagogy, as an indispensable component of $\mathrm{IaH}$, bears the responsibility of equipping students at these institutions with intercultural competence in order to give them the ability to communicate in the target language smoothly.

The present paper built upon data collected from a COIL exchange program, which aimed at bringing together learners from two universities in Japan and Taiwan, by video conferencing tools and helping them become aware of the cultural differences between the two countries. This was meant to enhance cross-cultural awareness among the learners and assist them to grow interculturally competent. Assessing the overall success of the program has not been a goal for this paper, however. The primary focus of this study was instead on how EFL learners engaged in online intercultural discussions who do not share an L1 manage to resolve communicative breakdowns in their interactions. The principal idea was that in such multicultural interactions since both the content and schemata might be unfamiliar to the learners, there is a higher possibility that the learners face communication difficulties. What the analysis of the data within a CA framework showed, however, was that although difficulties originating from gaps in the learners' English proficiency did exist, the participants managed to resolve them by switching the mode of their interaction from verbal to non-verbal (translingual and multimodal). This can have clear implications for foreign language curriculum designers and practitioners in tertiary education particularly in institutions where internationalization is a concern and learners have the opportunity to engage in multicultural exchanges.

First and foremost, the data suggests that a COIL-based curriculum can offer valuable opportunities for learners to experience intercultural interaction without having to bear the burden of traveling overseas. Learners can virtually experience being in a class with students from a different cultural background and discuss with them a wide range of cultural topics. This, of course, is by no means to undermine the benefits outbound student mobility can offer to an internationalization agenda. It is rather suggested as an alternative for outbound mobility where and when due to various constraints it is not possible for learners to experience IA.

Second, the conversation analysis of the data has shown that monolingual policies in foreign language education can no longer be tenable. Learners who share an L1 in an EFL setting resort to it as a semiotic recourse through translanguaging and those who do not share an L1 have a number of other semiotic resources at their disposal such as gaze, gesture, posture, referring and other non-verbal and non-linguistic resources.

Finally, it seems that language education in institutions with the primary concern of making learners interculturally aware and competent has to widen its perspective moving away from a narrow focus on the linguistic component to broader foci on interactional and intercultural components of the communicative competence.

Overall, it also seems that innovative curricula for foreign language pedagogy are needed to exploit the potentials computer technology and the internet can offer in order to cater for the emerging needs of learners who aspire to live as global citizens in a multilingual world. If intercultural interactions are multimodal by nature, learners may need to become aware of this feature and, if necessary, trained in it.

\section{REFERENCES}

Agnew, M. \& Kahn, H. E. (2014). Internationalizationat-home: Grounded practices to promote intercultural, international, and global learning. Metropolitan Universities, 25(3), 31-46.

Ally, M. (2004). Foundations of educational theory for online learning. In T. Anderson \& F. Elloumi (Eds.), Theory and practice of online learning (pp. 3-31). Athabasca, Canada: Athabasca University. 
Barraja-Rohan, A. M. (2011). Using conversation analysis in the second language classroom to teach interactional competence. Language Teaching Research, 15(4), 479-507. doi: $10.1177 / 1362168811412878$

Beelen J., \& Jones E. (2015) Redefining IaH. In Curaj A., Matei L., Pricopie R., Salmi J., Scott P. (Eds). The European Higher Education Area. Cham: Springer.

Button, G. \& Lee, J.R.E. (Eds.). (1987). Talk and social organisation. Clevedon: Multilingual Matters.

Canagarajah, S. (2017). Translingual practice as spatial repertoires: expanding the paradigm beyond structuralist orientations. Applied Linguistics, 39(1), 31-54. doi: 10.1093/applin/amx041

Cummins, J. (2007). Rethinking monolingual instructional strategies in multilingual classrooms. Canadian Journal of Applied Linguistics, 10(3), 221-240.

de Wit, H. (2010). Internationalization of higher education in Europe and its assessment, trends and issues. The Netherlands: NVAO.

Firth, A. \& Wagner, J. (2007). Second/foreign language learning as a social accomplishment: Elaborations on a reconceptualized SLA. The Modern Language Journal, 91(1), 800-819.

Have, P. T. (2012). Doing conversation analysis. Los Angeles: Sage.

Hawkins, M. (2018). Transmodalities and transnational encounters: Fostering critical cosmopolitan relations among global youth. Applied Linguistics, 39(1), 55-77.

Hudzik, J. (2011). Comprehensive internationalization: From concept to action. Washington: NAFSA.

Jefferson, G. (1984). Transcription Notation. In J. Atkinson and J. Heritage (Eds), Structures of Social Interaction. New York: Cambridge University Press.

Kääntä, L. (2012). Teachers' embodied allocations in instructional interaction. Classroom Discourse, 3(2), 166-186. doi: $10.1080 / 19463014.2012 .716624$

Kasper, G., \& Wagner, J. (2011). A conversationanalytic approach to second language acquisition. In D. Atkinson (Ed.), Alternative approaches to second language acquisition (pp. 117-142). New York: Taylor \& Francis.

Knight, J. (2004). Internationalization remodeled: Definition, approaches, and rationales. Journal of Studies in International Education, 8(1), 5-31.
Knight, J. (2006). Internationalization of higher education: New directions, new challenges. Paris: IAU.

Kramsch, C. (2002). Introduction: How can we tell the dancer from the dance? In C. Kramsch (Ed.), Language acquisition and language socialization: Ecological perspectives. London/New York: Continuum.

Kress, G. (2003). Literacy in the new media age. London: Routledge.

Mortensen, K. (2008). Selecting next speaker in the second language classroom: How to find a willing next speaker in planned activities. Journal of Applied Linguistics and Professional Practice, 5(1), 55-79. doi: 10.1558/japl.v5i1.55

Nor, N. F. M., Hamat, A. \& Embi, M. A. (2012) Patterns of discourse in online interaction: seeking evidence of the collaborative learning process. Computer Assisted Language Learning, 25(3), 237-256. doi: 10.80/09588221.2012.655748

OECD. (1996). Internationalizing the curriculum in higher education. Paris: OECD Publishing.

Pennycook, A. (1994). The cultural politics of English as an international language. London: Longman.

Roberts, C. \& Cook, M. (2009). Authenticity in the adult ESOL classroom and beyond. TESOL Quarterly, 43, 620-42.

Sacks, H., Schegloff, E. \& Jefferson, G. (1974). A simplest systematics for the organization of turntaking for conversation. Language, 50(4), 696-735.

Seedhouse, P. (2004). The interactional architecture of the language classroom: A conversation analysis perspective. Oxford: Blackwell.

Schegloff, E \& Sacks, H. (1973). Opening up closings. Semiotica, 8(4), 69-99.

Shiozaki, H. (2016). Collaborative Online International Learning (COIL) for Critical Reading of Media: Collaboration between IIUM and Kansai University. In: International Language and Tourism Conference 2016 (ILTC 2016), Kuala Lumpur.

Turnbull, M., \& Dailey-O Cain, J. (2009). First language use in second and foreign language learning. Bristol: Multilingual Matters.

Usó-Juan, E., \& Martínez-Flor, A. (2006). Current Trends in the Development and Teaching of the Four Language Skills. New York: Mouton de Gruyter.

Wagner, J. (2018). Multilingual and multimodal interactions. Applied Linguistics, 39(1), 99-107. 


\begin{tabular}{|c|c|}
\hline \multicolumn{2}{|c|}{ Appendix A: Guide to Transcription } \\
\hline JT & Japan class teacher \\
\hline TT & Taiwan class teacher \\
\hline $\mathrm{JL}$ & Japanese learner \\
\hline TL1-2 & Taiwanese learner 1 and 2 \\
\hline TLs & Taiwanese learners \\
\hline JLs & Japanese learners \\
\hline [ text ] & $\begin{array}{l}\text { Indicates the start and end points of } \\
\text { overlapping speech. }\end{array}$ \\
\hline$=$ & $\begin{array}{l}\text { Indicates the break and subsequent } \\
\text { continuation of a single interrupted } \\
\text { utterance. }\end{array}$ \\
\hline $\begin{array}{l}\text { (\# of } \\
\text { seconds) }\end{array}$ & $\begin{array}{l}\text { A number in parentheses indicates } \\
\text { the time, in seconds, of a pause in } \\
\text { speech. }\end{array}$ \\
\hline (.) & $\begin{array}{l}\text { A brief pause, usually less than } 0.2 \\
\text { seconds. }\end{array}$ \\
\hline . or $\downarrow$ & Indicates falling pitch. \\
\hline ? or $\uparrow$ & Indicates rising pitch. \\
\hline- & $\begin{array}{l}\text { Indicates an abrupt halt or } \\
\text { interruption in utterance. }\end{array}$ \\
\hline$>$ text $<$ & $\begin{array}{l}\text { Indicates that the enclosed speech } \\
\text { was delivered more rapidly than } \\
\text { usual for the speaker. }\end{array}$ \\
\hline$<$ text $>$ & $\begin{array}{l}\text { Indicates that the enclosed speech } \\
\text { was delivered more slowly than } \\
\text { usual for the speaker. }\end{array}$ \\
\hline$\circ$ & $\begin{array}{l}\text { Indicates whisper or reduced } \\
\text { volume speech. }\end{array}$ \\
\hline ALL CAPS & $\begin{array}{l}\text { Indicates shouted or increased } \\
\text { volume speech. }\end{array}$ \\
\hline$:::$ & $\begin{array}{l}\text { Indicates prolongation of an } \\
\text { utterance. }\end{array}$ \\
\hline (hhh) & Audible exhalation \\
\hline ? or (.hhh) & Audible inhalation \\
\hline ( text ) & $\begin{array}{l}\text { Speech which is unclear or in } \\
\text { doubt in the transcript. }\end{array}$ \\
\hline $\begin{array}{l}((\text { italic } \\
\text { text }))\end{array}$ & Annotation of non-verbal activity. \\
\hline
\end{tabular}




\section{APPENDIX B: Screenshots}

Screenshot 1

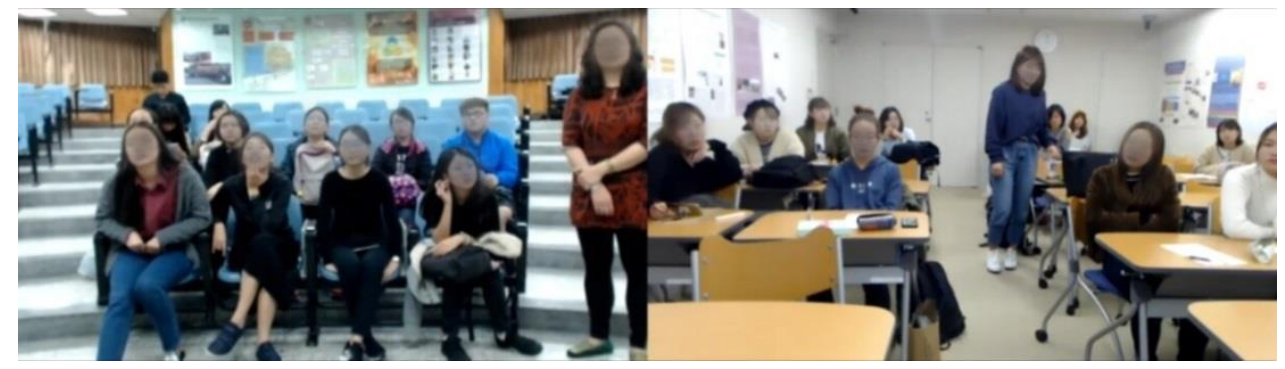

Screenshot 2

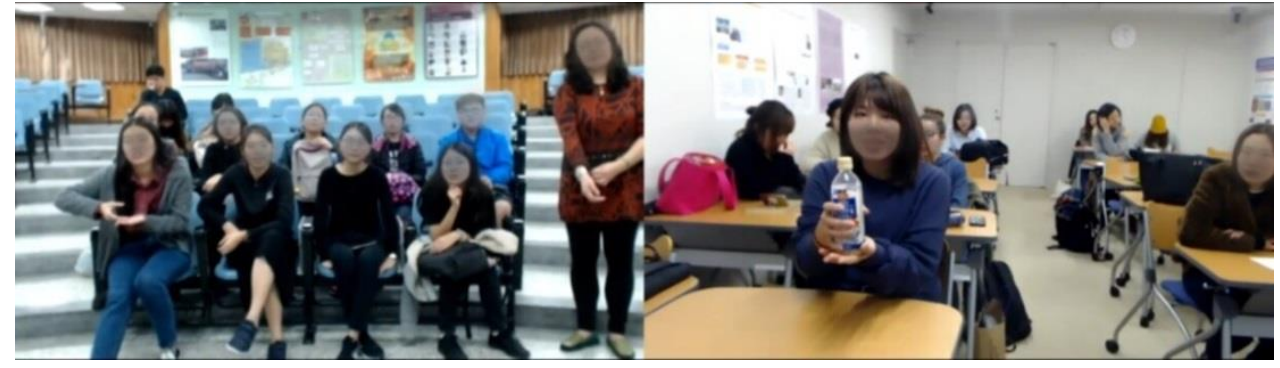

Screenshot 3

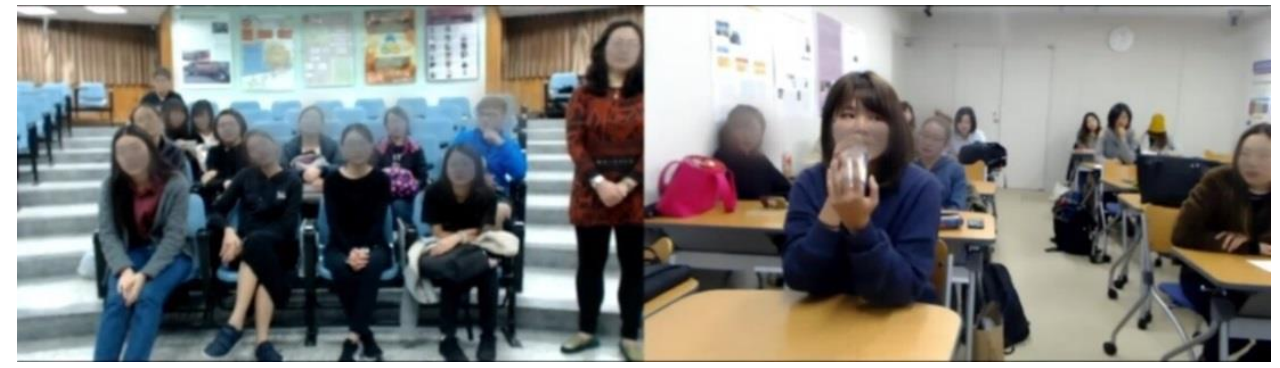

Screenshot 4

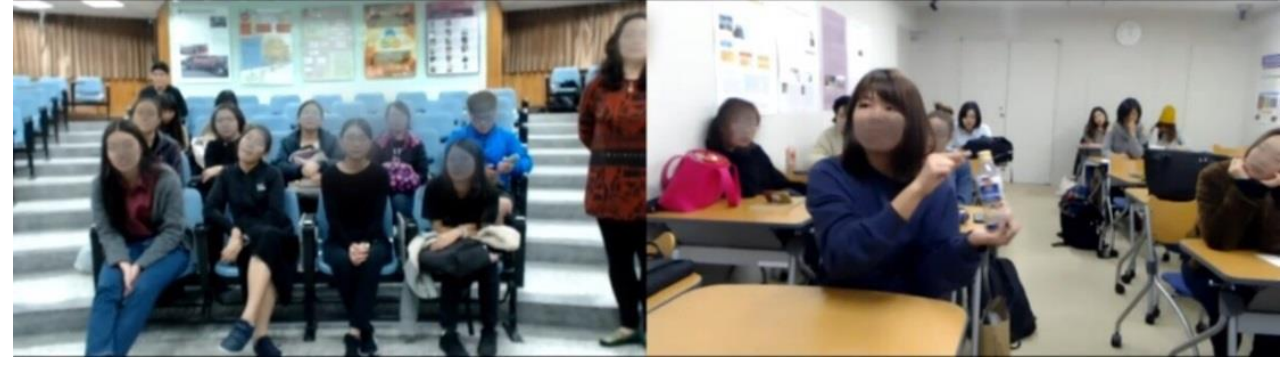

Screenshot 5

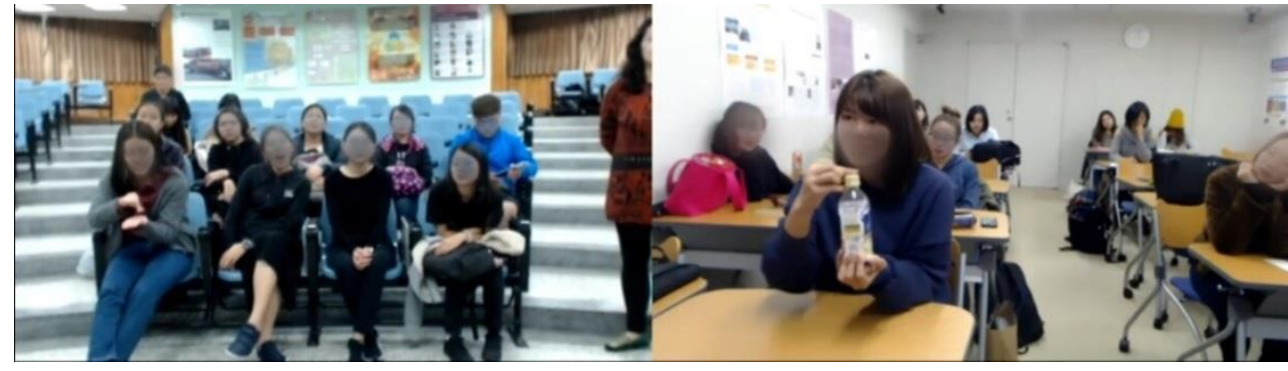

Copyright @ 2018, IJAL, e-ISSN: 2502-6747, p-ISSN: 2301-9468 